\title{
A Transmitter Architecture for Nonconstant Envelope Modulation
}

\author{
C. Berland, Member, IEEE, I. Hibon, J. F. Bercher, M. Villegas, D. Belot, D. Pache, and V. Le Goascoz
}

\begin{abstract}
This paper presents a transmitter architecture which is less sensitive to power amplifier AM/AM and AM/PM conversions. Indeed, most radio communication standards employ nonconstant envelope modulation such as in the 802.11 series. Owing to power amplifiers' nonlinearities, distortions on the modulations degrade the overall transmitter performance. As a consequence, linearization methods, such as envelope elimination and restoration, have to be employed. The architecture relies on the transformation of a nonconstant envelope modulation to a constant or switched one. This signal coding enables the efficient amplification of the signal without undergoing power amplifier distortions. The envelope variation is restored by bandpass filtering just after the power amplifier.
\end{abstract}

Index Terms-Linearization techniques, pulsewidth modulation (PWM), sigma-delta $(\Sigma \Delta)$ modulation, transceivers.

\section{INTRODUCTION}

$\mathbf{E}$ NVELOPE variation in digital modulation increases transmitter design complexity. AM/AM and AM/PM conversion effects of the power amplifier (PA) degrade error vector magnitude (EVM) and the output spectrum of the transmitted signal. Therefore, linearization methods, one of which is envelope elimination and restoration (EER), have to be implemented. EER [1] is an analog solution that relies upon the separation of the envelope and phase parts of the modulated signal. The envelope signal is restored through its injection via the PA supply voltage. Pulsewidth modulation (PWM) of the envelope and amplification via a Class-D amplifier achieve this operation, as demonstrated in Fig. 1. The dc-dc converter implementation is complex because of the envelope variation and bandwidth it has to handle.

Advances in technologies, together with the significant improvement in the frequency capabilities of heterojunction bipolar and CMOS, now enable the design of high frequency switched circuits, e.g a $8-\mathrm{GHz}$ sigma-delta $(\Sigma \Delta)$ [2], or a 16-GHz comparator [3].

These advances allow the classical EER architecture to be adapted using such high-frequency (HF) circuits to produce a new solution which is more efficient and has beneficial characteristics.

Manuscript received September 14, 2004; revised February 16 2005. This paper was recommended by Associate Editor F. C. M. Lau.

C. Berland, J. F. Bercher, and M. Villegas are with ESYCOM-ESIEE laboratoire système de communication, 93162 Noisy-Le-Grand Cedex, France (e-mail: c.berland@esiee.fr).

I. Hibon, D. Belot, D. Pache, and V. Le Goascoz are with ST Microelectronics, 38926 Crolles Cedex, France (e-mail: isabelle.hibon@st.com).

Digital Object Identifier 10.1109/TCSII.2005.854594

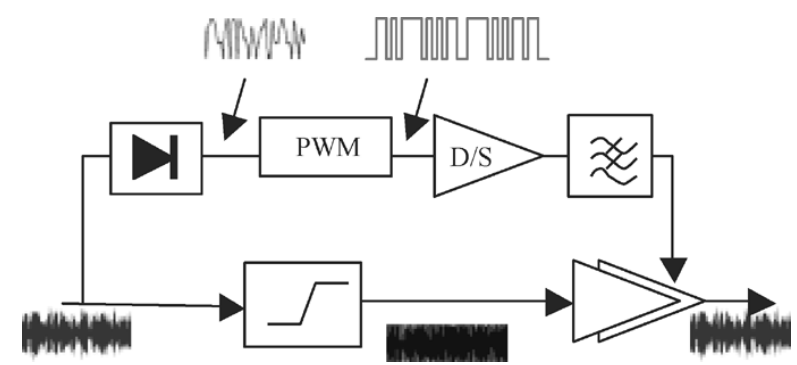

Fig. 1.PWM and restoration of the envelope.

The classical EER architecture can be modified in such a way that the restoration occurs before the power amplification by a simple multiplication between the constant envelope phase signal and that of the PWM. The envelope variation is recovered after the power amplifier by bandpass filtering. Such an architecture using a " $0 / 1 \Sigma \Delta$ " was proposed recently in [4]. Another development of the original EER is given in [5] and presented here. In order to avoid degradations related to the envelope detector and to the limiter, we choose to generate digitally both phase and envelope signals, as indicated in Fig. 2.

A significant improvement is obtained using a " $\pm a \Sigma \Delta$ " which results in a constant envelope signal at the PA input as shown in Fig. 3. Consequently, the choice of PA is not reduced to the single switched PA class. This solution also simplifies the implementation of the PA dc-dc converter.

This paper presents performances of this new transmitter architecture applied to a 16 QAM and an orthogonal frequency-division multiplexing (OFDM) modulation. The discussion will be mainly focused on the PWM generation (classical in Section III and using a 1-bit " $\pm a \Sigma \Delta$ " in Section IV) and its impact on characteristics of other transmitter components. In both cases, we present the architecture and related properties, its tuning, and its performances. Finally, the conclusion highlights the benefits of this approach.

\section{PRELIMINARY - InTRODUCTION TO OFDM MODULATION}

Due to its properties in terms of bandwidth and envelope magnitude variation, the OFDM modulation of hiperlan2 standard is chosen here to validate the transmitter architecture. The transmission frequency is about $5 \mathrm{GHz}$, with a 16-QAM symbol time duration of $50 \mathrm{~ns}$. For comparison purposes, the architecture is also studied in the case of a simple 16-QAM modulation with the same symbol time duration.

The OFDM principle consists in the multiplication of subcarriers with QAM symbols. The time-domain signal which is 


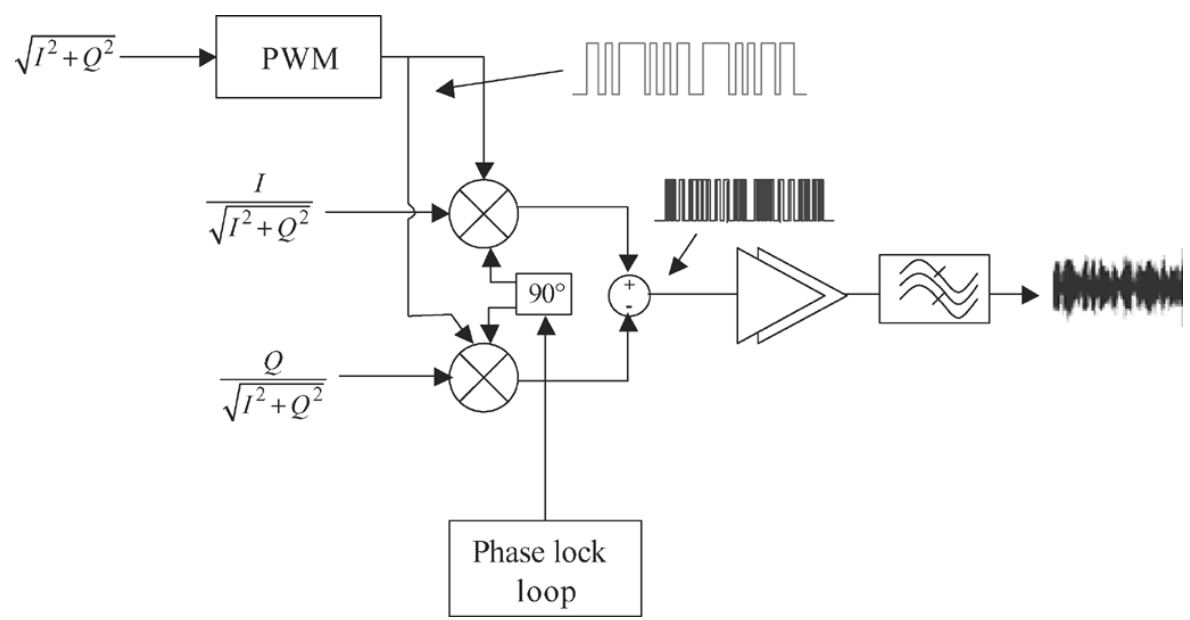

Fig. 2. Principle of the new transmitter architecture.

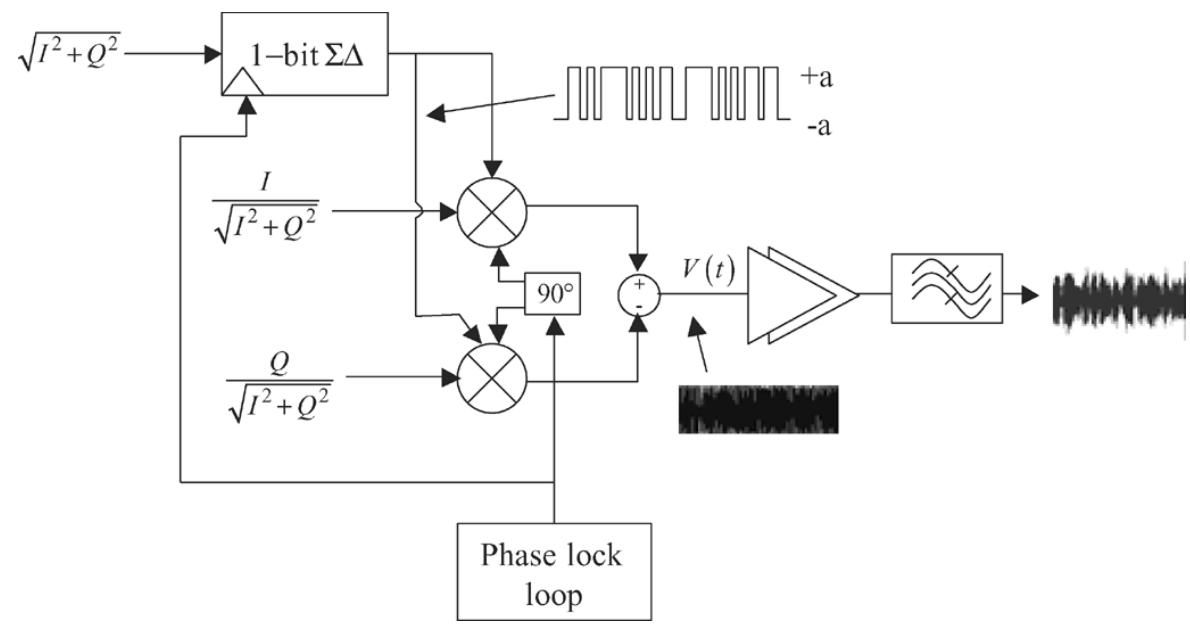

Fig. 3. New transmitter architecture with a 1-bit $\Sigma \Delta$ modulator.

obtained after inverse fast Fourier transform can be written as follows:

$$
s(t)=\sum_{k} \sum_{n} a_{k, n} \cos \left(\omega_{n} t+\varphi_{k, n}\right) .
$$

The OFDM signal presents large magnitude variations, as demonstrated with 64 subcarriers in Fig. 4, since the difference between the maximum and the mean power is up to $18 \mathrm{~dB}$, while this difference is only of $6 \mathrm{~dB}$ for the classical 16 QAM realized with a raised cosine filter with a 0.5 roll off.

As a consequence, this modulation is highly sensitive to power amplifier AM/AM and AM/PM distortions. Bandwidths of the envelope and phase signals are also large, the former above $60 \mathrm{MHz}$ and the latter above $80 \mathrm{MHz}$ [6]. Such characteristics would tighten the transmitter's specification and so point up its limitations in comparison with the 16 QAM.

\section{TRAnSmitTER ARChitecture With Classical PWM GenERATION}

\section{A. PWM Envelope Coding}

The first method used for the PWM generation is based on the comparison between the signal and a sawtooth or triangle shape as presented in Fig. 5.


Fig. 4. Envelope magnitude variation for a 64 sub-carrier OFDM in comparison with a 16 QAM.

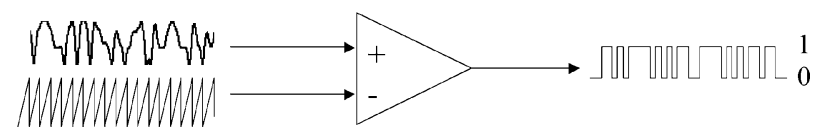

Fig. 5. PWM using a sawtooth signal and an operational amplifier.

The output signal is then a series of pulses with " 0 " or " 1 " values. The result of its multiplication with the phase signal, see 


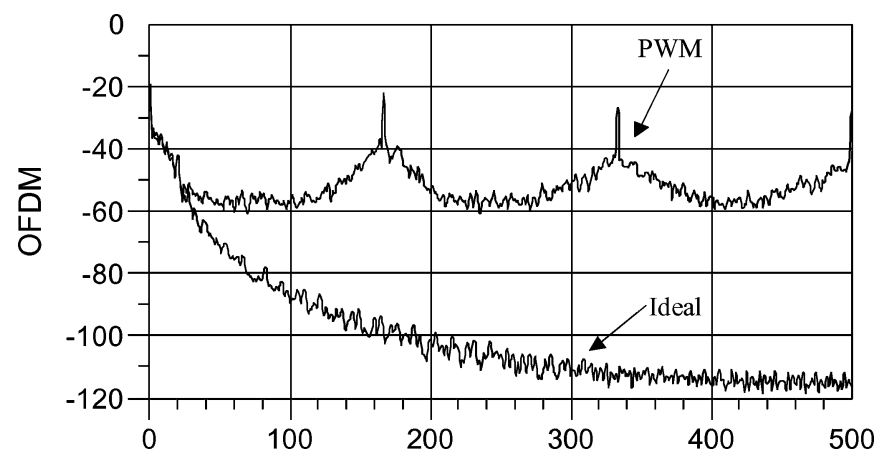

Fig. 6. Power spectral density of the PWM coded OFDM envelope using a $180-\mathrm{MHz}$ saw_tooth reference signal by comparison with the ideal OFDM envelope signal PSD.



Fig. 7. Ideal and reconstructed signal spectrums for OFDM modulation.

Fig. 2, is then amplified for instance with a Class-C or Class-E switched amplifier.

An important drawback of this method is the high level of intermodulation terms. When applied to an envelope signal, this method generates harmonics of the reference signal and mixing products as presented in Fig. 6. For both modulation schemes, the optimum shape and frequency of the reference signal have to be found to reduce spurious levels. These optima are selected as an heuristic tradeoff between the noise level, the locations of peaks and the transmitter EVM. They are consistent with theoretical results of [7]. For the 16 QAM, the optimum shape is a 200-MHz sawtooth, whereas for the OFDM modulation, although the optimum shape is the same, the frequency was lowered to $180 \mathrm{MHz}$. Increasing the reference frequency enlarges intermodulation terms and degrades transmitter EVM (8\% with a 1-GHz sawtooth).

\section{B. Transmitter Performances}

Fig. 7 presents the signal spectrum at the input of the power amplifier obtained from the multiplication of the PWM envelope signal with the phase signal and the local oscillator. We find the spectrum of Fig. 6, shifted in frequency and reconstructed with the phase signal so that the central part is an real OFDM signal.

This method raises two difficulties: the output filter bandwidth and the EVM value.

As far as the first difficulty is concerned, radio-communication standards always require spectrum template and low level of spurious emissions. With the presence of mixing products and high reference harmonics, spurious emissions out of the channel bandwidth are at least $-30 \mathrm{dBc}$ in $1-\mathrm{MHz}$ bandwidth.

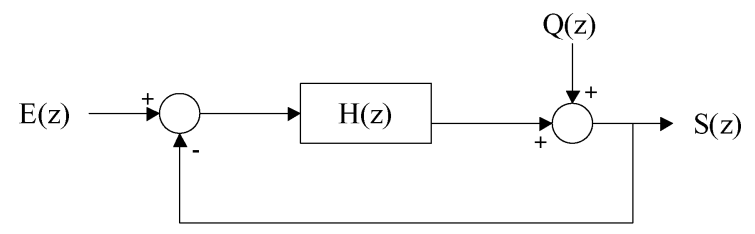

Fig. 8. Linearized model of a $\Sigma \Delta$ modulator.

If the standard specifies that the level has to be lower, the output filter would have to have a low relative bandpass: in our example this corresponds to $0.4 \%$ for a $5-\mathrm{GHz}$ carrier. The second difficulty concerns the value of the ideal EVM value: $1.5 \%$ for the 16 QAM but $3.2 \%$ for the OFDM modulation. For the latter, when a Class-E power amplifier is added into the simulation, the EVM reaches $4 \%$ but with no degradation of the output spectrum.

With additional component imperfections, such as I/Q modulator mismatch and synthesizer phase noise both impacting EVM of about 5\%, the global EVM value is too high for radiocommunication systems [6]. This implies that the limits of this solution have been reached.

\section{TRAnSmitter ARChiteCtURE USING A 1 -BIT $\Sigma \Delta$ MODULATOR}

In this section, we show that the use of a " $\pm a \Sigma \Delta$ " ends with a constant envelope signal at the input of the PA. This key point will result in a highly linear and efficient transmitter.

\section{A. Constant Envelope Property}

An alternative to classical PWM coding is the use of a 1-bit $\Sigma \Delta$ modulator, see Fig. 3, in which the quantified noise is moved out of band. The aim of its design is then to reject the noise out of the envelope bandwidth in such a way that it releases the output filter bandwidth. After the multiplication between the 1-bit $\Sigma \Delta$ output, the phase signals and the local oscillator, we obtain:

$$
\begin{aligned}
V(t) & = \pm a \cdot(A \cos (\omega t) \cos \varphi-A \sin (\omega t) \sin \varphi) \\
& = \pm a \cdot A \cos (\omega t+\varphi) \\
& =A a \cos (\omega t+\varphi \pm \pi)
\end{aligned}
$$

which is the expression of a constant envelope signal.

In comparison with the former PWM solution or with the " $0 / 1$ $\Sigma \Delta$ " solution of [4], the power amplification is highly simplified so that any type of amplifier can fit this transmitter since its input signal has a constant envelope (the 0/1 solution may require a specifically designed linear amplifier optimized for establishment time).

\section{B. $\Sigma \Delta$ Architecture and Envelope Coding}

The linearized model of a 1-bit $\Sigma \Delta$ is given in Fig. 8 .

The output signal can then simply be written as follows:

$$
S(z)=\frac{H(z)}{H(z)+1} E(z)+\frac{1}{H(z)+1} Q(z)
$$

with $Q(z)$ the quantization noise.

The first term of this equation corresponds to the signal transfer function (STF) and the second part corresponds to the 




Fig. 9. $A \Sigma \Delta$ feedback structure with optional local feedback.

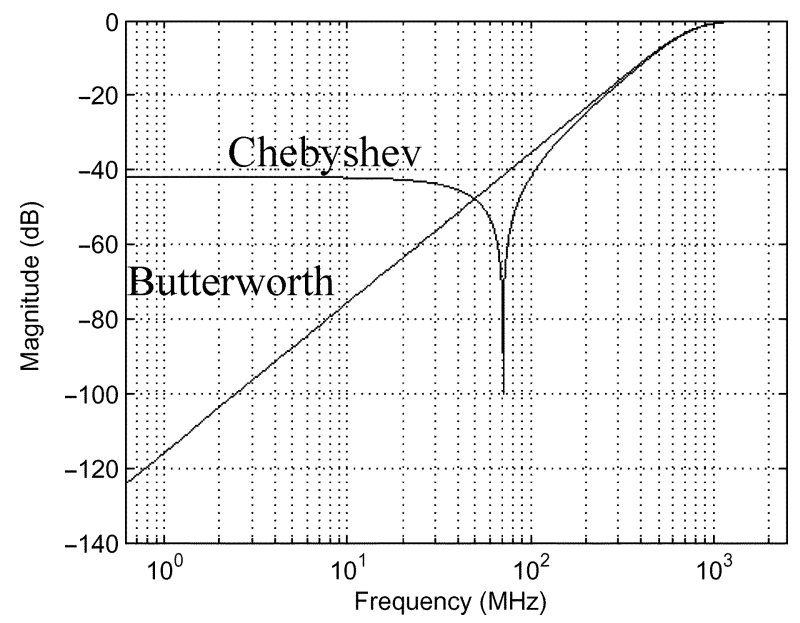

Fig. 10. Theoretical NTF of 1-bit second-order $\Sigma \Delta$ with Butterworth and inverse Chebyshev structures.

noise transfer function (NTF), both dependent on $H(z)$. In the architecture, the $\Sigma \Delta$ is devised so that the former is a low pass filter and the latter a high pass one. This is achieved with a structure such as the one in Fig. 9, where, depending on the presence of the local feedback b1, a third-order Butterworth or inverse Chebyshev NTF can be obtained.

In our application, we aimed to limit the noise floor at the transmitter output below $-40 \mathrm{dBc}$, in $1-\mathrm{MHz}$ measurement bandwidth, for an output bandwidth of $200 \mathrm{MHz}$. In order to reach this objective, we calculated several $\Sigma \Delta$ with a minimum bandwidth of $100 \mathrm{MHz}$. Such a bandwidth can only be realized with a high sampling frequency and to achieve this, we took advantage of the local oscillator to drive the $\Sigma \Delta$.

As the sampling frequency and the bandwidth are imposed, the oversampling ratio (OSR) is

$$
\mathrm{OSR}=\frac{F_{s}}{2 * B w}=\frac{5.10^{9}}{200.10^{6}}=25
$$

With the OSR fixed by the architecture, the only remaining parameters which can be varied are the order and structure of the $\Sigma \Delta$ [8]. The determination and choice of this order and structure must take into account complexity of the design, stability, and the signal to noise ratio. In order to examine these points, four 1-bit $\Sigma \Delta$ were studied: two second-order ones, whose theoretical NTF are presented in Fig. 10, and two third-order ones, all with Butterworth or inverse Chebyshev structure. The major difficulty of their design concerned stability. The structure coefficients were established by adjusting them to the theoretical high pass transfer function needed for the application. Nevertheless, according to the value of the linearizedquantizer gain,
TABLE I

$\Sigma \Delta$ SUMMARIZED PERFORMANCES

\begin{tabular}{c|c|c|c|c}
\hline & $2^{\text {nd }}$ Butt & $2^{\text {nd }}$ Cheb & $3^{\text {rd }}$ Butt & $3^{\text {rd }}$ Cheb \\
\hline NTF gain & 1.93 & 1.95 & 1.61 & 1.69 \\
\hline Input level (dB) & -11.8 & -11.4 & -14.5 & -15.5 \\
\hline SNR (dB) & 44.6 & 46.8 & 46.5 & 54.0 \\
\hline $\begin{array}{l}\text { Noise (dB) } \\
\text { at } 100 \mathrm{MHz}\end{array}$ & -70 & -80 & -70 & -90 \\
\hline
\end{tabular}

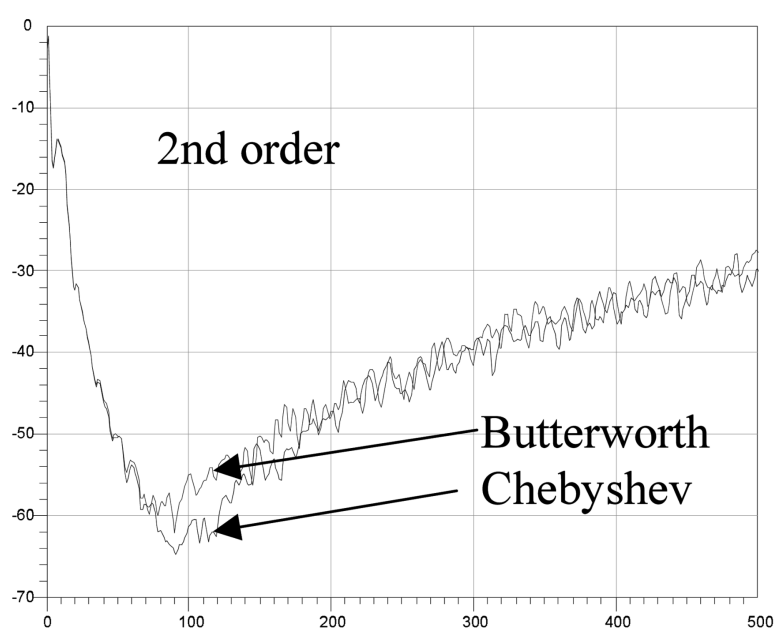

Fig. 11. 1-bit second-order $\Sigma \Delta$ modulator output spectra with an OFDM envelope signal as an input for Butterworth and Chebyshev structure.

which depends on the input signal level, instability may occur. This was evaluated using a root locus technique [9] that enables the specification of the maximum stable input signal level. A second criterion to avoid instability is to keep the $\Sigma \Delta$ NTF gain below 2 [10].

Table I summarizes the four $\Sigma \Delta$ performances, in the case of a sinusoidal input, with the following parameters: NTF gain, the input signal level relative to a feedback signal equal to 1 , the SNR in 100-MHz bandwidth and the noise level at $100 \mathrm{MHz}$ (relative to a feedback signal equal to 1 ).

For all $\Sigma \Delta$, the noise level at $F_{s} / 2$ remain under $-20 \mathrm{~dB}$ relative to a feedback signal equal to 1 .

As expected, the SNR increases between the Butterworth and inverse Chebyshev structures. This gain is about $2 \mathrm{~dB}$ for a second-order $\Sigma \Delta$ and more than $7 \mathrm{~dB}$ for the third-order one. However, when the two Butterworth structures are compared, the SNR amelioration is insufficient in view of the increase in the complexity of the structure.

The previous analysis was done with a pure sinusoidal input. Therefore, results and conclusions may be quite different with ODFM or 16-QAM envelope signal. The envelope signal is applied in such a way that its mean level corresponds to the equivalent sinusoidal signal indicated in Table I so that the relative maximum input level never exceeds the maximum stable signal. Fig. 11 presents 1-bit $\Sigma \Delta$ modulator output spectra with the ODFM modulation for the two second-order configurations. The SNR improvement between the two structures, whatever the order, is about $5 \mathrm{~dB}$. Likewise, the same result is found when comparing the second and third order. 
TABLE II

RMS EVM PERFORMANCES

\begin{tabular}{l|c|c|c|c}
\hline & $2^{\text {nd }}$ Butt & $2^{\text {nd }}$ Cheb & $3^{\text {rd }}$ Butt & $3^{\text {rd }}$ Cheb \\
\hline 16 QAM & $0.46 \%$ & $0.52 \%$ & $1.78 \%$ & $1.36 \%$ \\
\hline OFDM & $0.35 \%$ & $0.40 \%$ & $1.26 \%$ & $0.78 \%$ \\
\hline
\end{tabular}

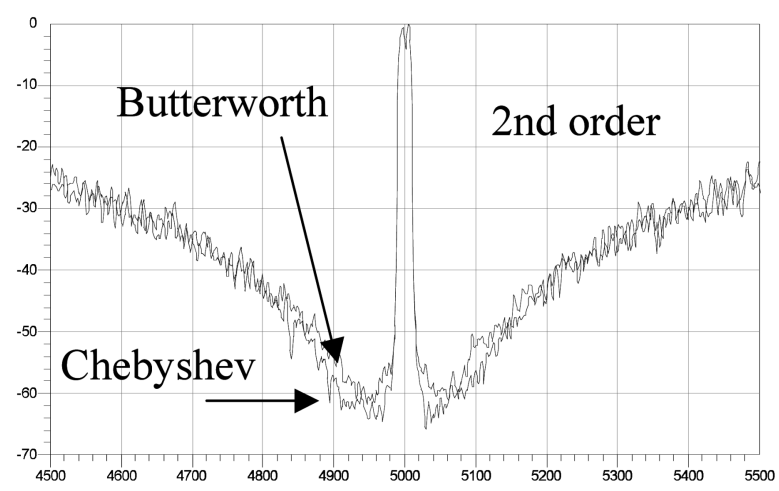

Fig. 12. Output spectra with an OFDM modulated signal for second-order Butterworth and Chebyshev $\Sigma \Delta$.

\section{Transmitter Performances}

The real impact of the $\Sigma \Delta$ choice has to be evaluated on the global transmitter architecture after the bandpass filter. In our simulations, to avoid distortions linked to variations of propagation time, we chose a raised cosine filter with $200-\mathrm{MHz}$ bandwidth.

The output signal is formed by the multiplication of envelope and phase signals, which corresponds to a convolution in the spectral domain. Although higher order $\Sigma \Delta$ have better noise performances inside the signal bandwidth, the out of band noise shape presents a steeper slope. As a result, the convolution between the phase signal and the out of band noise produces a deterioration in the SNR which more than offsets the improvement linked to $\Sigma \Delta$ order increase, as demonstrated in Table II with rms EVM values.

In comparison with classical PWM architecture, the EVM results are better: $0.35 \%$ with OFDM modulation and secondorder Butterworth $\Sigma \Delta$ instead of $3.2 \%$. Concerning the " $0 / 1$ " $\Sigma \Delta$, simulations for OFDM modulation showed a high EVM ( $>11 \%)$ due to bad envelope restoration.

The $\pm a \Sigma \Delta$ architecture using an OFDM modulation was also simulated with a Class-B power amplifier and gives an EVM value of $0.38 \%$ with just slight modifications of the output spectrum.

It is also important to note that this architecture is less sensitive to the modulation type than the previous one. Moreover, the output spectra of OFDM and 16 QAM are very similar and both respect the $-40 \mathrm{dBc}$ constraint in a $200-\mathrm{MHz}$ bandwidth. Concerning the spectrum to be filtered, see Fig. 12, the absence of harmonics makes it easier to filter and generates less disturbance on the radio part. The output filter would have to keep its filtering property on a large bandwidth. As the quantization noise is at the input of the power stage, the SNR remains constant whatever the output power.

The efficiency of this architecture relies mainly upon the $\Sigma \Delta$ consumption and the output power. At high frequency, the $\Sigma \Delta$ will consume few hundred milliwatts (a 4-GHz $\Sigma \Delta$ in [11] consumes $350 \mathrm{~mW}$ ). In the Hiperlan2 case, PA are usually used at 6 dB back-off (Anadigics, RFS P5022), which decreases their efficiency from $56 \%$ to $14 \%$. For a $1-\mathrm{W}$ transmitter, the architecture presented could reach $45 \%$ efficiency with the $\Sigma \Delta$ in [11] and the same PA working at its 1-dB compression point. Clearly, using a more efficient PA such as Class E, the efficiency will be even greater.

\section{CONCLUSION}

This paper presents a new versatile digital transmitter architecture that can be adopted for any non constant modulation, on a wide range of frequencies, with high linearity and efficiency. This architecture is an improvement of the classical EER architecture, and consists in PWM coding the envelope of a modulated signal. Two methods for the PWM generation are analyzed: the simplest method transforms the modulated signal into a switched one. This generates harmonics and intermodulation terms, so this architecture may present insufficient performances for some modulation schemes. The second solution uses a " $\pm a \Sigma \Delta$ " modulator and enables the transformation of any modulated signal into a constant envelope one. This requires a high frequency 1-bit $\Sigma \Delta$ modulator that can be devised either in digital CMOS or BiCMOS technologies, according to the sampling frequency.

Even with severe modulations such as OFDM, this solution gives the desired level of performance in terms of EVM and output spectra. The other advantage compared to classical EER concerns the dc-dc converter which would be easier to implement.

\section{REFERENCES}

[1] L. Kahn, "Single sideband transmission by envelope elimination and restoration," in Proc. IRE, Jul. 1952, pp. 803-806.

[2] S. Krishnan and D. Scott et al., "An 8-GHz continuous-time $\Sigma-\Delta$ analog-digital converter in an InP-based HBT technology," IEEE Trans. Microw. Theory Tech., vol. 51, no. 12, Dec. 2003.

[3] J. C. Jensen and L. E. Larson, "A 16-GHz Yltra-high-speed Si-SiGe HBT comparator," IEEE J. Solid-State Circuits, vol. 38, no. 9, pp. [AU: PAGE NUMBERS?]-, Sep. 2003.

[4] Y. Wang, "An improved Kahn transmitter architecture based on delta-sigma modulation," in Proc. IEEE Microw. Symp., Philadelphia, PA, Jun. 2003.

[5] C. Berland, J. F. Bercher, I. Hibon, M. Villegas, D. Belot, D. Pache, and V. Le Goascoz, "Dispositif de modulation et émetteur comprenant un tel dispositif," French Patent FR0 405 636, May 25, 2004.

[6] G. Baudoin, C. Berland, M. Villegas, and A. Diet, "Influence of time and processing mismatches between phase and envelope signals in linearization systems using envelope elimination and restoration, application to Hiperlan2," in Proc. IEEE Microw. Symp., Philadelphia, PA, Jun. 8-13, 2003.

[7] E. R. Kretzmer, "Distortion in pulse-duration modulation," Proc. IRE, pp. 1230-1235, Nov. 1947.

[8] S. R. Norsworthy, R. Schreier, and G. C. Temes, Delta-Sigma Data Converters. Theory, Design, and Simulation. New York: IEEE Press, 1997, ch. 3 .

[9] A. Gothenberg, B. Li, and H. Tenhunen, "A method for stability and performance analysis of low oversampling ratio higher order sigma-delta noise shaper architectures," in Proc. IEEE 42nd Midwest Symp. Circuits Syst., vol. 1, 1999.

[10] K. C. H. Chao, S. Nadeem, W. L. Lee, and C. G. Sodini, "A higher order topology for interpolative modulators for oversampling A/D converters," IEEE Trans. Circuits Syst., vol. 37, no. 3, pp. 309-318, Mar. 1990.

[11] W. Gao, J. A. Cherry, and W. N. Snelgrove, "A 4-GHz fourth-order SiGe HBT band pass $\Delta \Sigma$ modulator," in Dig. Tech. Papers Symp. VLSI Circuits, Jun. 11-13, 1998, pp. 174-175. 